\title{
NEW RELATIONS BETWEEN DIS SUM RULES
}

\author{
A. L. KATAEV* \\ Institute for Nuclear Research of the Academy of Sciences of Russia, Moscow, 117312, Russia \\ *E-mail: kataev@ms2.inr.ac.ru
}

\begin{abstract}
New relations between Bjorken polarized, Gross-Llewellyn Smith and Bjorken unpolarized sum rules are proposed. They are based on the "universality" of the perturbative and non-perturbative $1 / \mathrm{Q}^{2}$ contributions to these sum rules. The letter facts can be deduced from the corresponding renormalon calculations. The similarity of $1 / \mathrm{Q}^{2}$ corrections are checked by inspecting the numerical results obtained within several approaches. The discussed relations are in agreement with existing experimental data.Some possible new phenomenological applications are mentioned including estimates of not yet measured Bjorken unpolarized sum rule.
\end{abstract}

Keywords: deep-inelastic sum rules, perturbative and non-perturbative effects of QCD

There are approximate relations between the Bjorken sum rule for polarized charged leptons-nucleon DIS (Bp), the Gross-Llewellyn Smith sum rule (GLS) and Bjorken unpolarized sum rule (Bup) for $\nu N$ DIS ${ }^{1},{ }^{2}$, namely

$$
\operatorname{Bp}\left(\mathrm{Q}^{2}\right) \approx \frac{\mathrm{g}_{\mathrm{A}}}{18} \mathrm{GLS}\left(\mathrm{Q}^{2}\right) \approx \frac{\mathrm{g}_{\mathrm{A}}}{6} \mathrm{Bup}\left(\mathrm{Q}^{2}\right)
$$

They include perturbative QCD corrections and non-perturbative $1 / \mathrm{Q}^{2}$-effects and are valid in the case when $\mathrm{O}\left(1 / \mathrm{Q}^{4}\right)$ terms can be neglected. Eq.(1) was discovered by analysing the renormalon model calculations of Refs. 3, 4. The definitions of the sum rules we are interested in are well-known:

$$
\begin{aligned}
\mathrm{GLS} & =\frac{1}{2} \int_{0}^{1}\left[\mathrm{~F}_{3}^{\nu \mathrm{n}}\left(\mathrm{x}, \mathrm{Q}^{2}\right)+\mathrm{F}_{3}^{\nu \mathrm{p}}\left(\mathrm{x}, \mathrm{Q}^{2}\right)\right] \mathrm{dx}, \\
\mathrm{Bp} & =\int_{0}^{1}\left[\mathrm{~g}_{1}^{\operatorname{lp}}\left(\mathrm{x}, \mathrm{Q}^{2}\right)-\mathrm{g}_{1}^{\ln }\left(\mathrm{x}, \mathrm{Q}^{2}\right)\right] \mathrm{dx}, \\
\text { Bup } & =\int_{0}^{1}\left[\mathrm{~F}_{1}^{\nu \mathrm{p}}\left(\mathrm{x}, \mathrm{Q}^{2}\right)-\mathrm{F}_{1}^{\nu \mathrm{n}}\left(\mathrm{x}, \mathrm{Q}^{2}\right)\right] \mathrm{dx} .
\end{aligned}
$$

Within QCD they can be expressed as

$$
\begin{aligned}
\operatorname{GLS}\left(\mathrm{Q}^{2}\right) & =3\left[\mathrm{C}_{\mathrm{GLS}}\left(a_{s}\right)-\frac{\mathrm{A}}{\mathrm{Q}^{2}}+\mathrm{O}\left(1 / \mathrm{Q}^{4}\right)\right] \\
\mathrm{Bp}\left(\mathrm{Q}^{2}\right) & =\frac{\mathrm{g}_{\mathrm{A}}}{6}\left[\mathrm{C}_{\mathrm{Bp}}\left(a_{s}\right)-\frac{\mathrm{B}}{\mathrm{Q}^{2}}+\mathrm{O}\left(1 / \mathrm{Q}^{4}\right)\right] \\
\operatorname{Bup}\left(\mathrm{Q}^{2}\right) & =\mathrm{C}_{\mathrm{Bup}}\left(a_{s}\right)-\frac{\mathrm{C}}{\mathrm{Q}^{2}}+\mathrm{O}\left(1 / \mathrm{Q}^{4}\right)
\end{aligned}
$$

where $a_{s}=a_{s}\left(\mathrm{Q}^{2}\right)=\alpha_{s}\left(\mathrm{Q}^{2}\right) / \pi$ and the coefficient functions

$$
\begin{aligned}
\mathrm{C}_{\mathrm{GLS}}\left(a_{s}\right) & =1-4 a_{s}-O\left(a_{s}^{2}\right) \\
\mathrm{C}_{\mathrm{Bp}}\left(a_{s}\right) & =1-4 a_{s}-O\left(a_{s}^{2}\right) \\
\mathrm{C}_{\mathrm{Bup}}\left(a_{s}\right) & =1-\frac{8}{3} a_{s}-O\left(a_{s}^{2}\right)
\end{aligned}
$$

are explicitly calculated up to $a_{s}^{3}$-corrections (for a review see ${ }^{5}$ ). The non-perturbative parameters $\mathrm{A}$ and $\mathrm{C}$ of the $1 / \mathrm{Q}^{2}$ corrections to the $\nu N$ DIS sum rules are connected to matrix elements of operators composed from quark and gluon fields, and written down in Ref. 6. The numerator $\mathrm{B}$ of the $1 / \mathrm{Q}^{2}$ term in the $\mathrm{Bp}$ sum rule is defined by matrix elements calculated in Ref.7, with additional input from Ref.8. The discussions presented below are mainly based on the work ${ }^{2}$.

The "universality" of perturbative contributions to the sum rules means that the asymptotic structures of the expansion of their coefficient functions in the QCD coupling constant is almost identical, namely

$$
\mathrm{C}_{\mathrm{GLS}}\left(a_{s}\right) \approx \mathrm{C}_{\mathrm{Bp}}\left(a_{s}\right) \approx \mathrm{C}_{\mathrm{Bup}}\left(a_{s}\right) .
$$

The "universality" of the non-perturbative $1 / \mathrm{Q}^{2}$-contributions to the same sum rules implies that

$$
\mathrm{A} \approx \mathrm{B} \approx \mathrm{C}
$$


Following 2 , consider now the method of renormalon calculus, advanced in Ref. ${ }^{9}$, and reviewed in detail in 10 and ${ }^{11}$. The coefficient function of DIS sum rules can be presented in the form of the Borel integral

$$
\mathrm{C}\left(a_{s}\right)=\int_{0}^{\infty} \exp \left(-\delta / \beta_{0} a_{s}\right) \mathrm{B}[\mathrm{C}(\delta)] \mathrm{d} \delta
$$

where $\mathrm{B}[\mathrm{C}(\delta)]$ is the Borel transform, defined as $\mathrm{B}[\mathrm{C}(\delta)]=\sum_{n=0}^{\infty} \mathrm{d}_{\mathrm{n}}\left(\delta^{\mathrm{n}} / \mathrm{n} !\right)$. Here $\mathrm{d}_{\mathrm{n}}$ are the coefficients of the asymptotic perturbative expansion of $\mathrm{C}\left(a_{s}\right)$ and $\beta_{0}=(11 / 3) C_{A}-$ $(4 / 3) T_{f} N_{f}$ is the first coefficient of the QCD $\beta$-function, $C_{A}=3, T_{f}=1 / 2$ and $N_{f}$ - number of the active quarks flavours. The usual prescription of calculating the Borel transform in QCD is to evaluate the contributions of Feynman diagrams with one gluon line, dressed by a chain of fermion loops, each proportional to $N_{f}$. Their contributions to the coefficient functions do not reflect the whole picture of renormalon effects in QCD. The latter begin to manifest themselves after the replacement $N_{f} \rightarrow-(3 / 2) \beta_{0}=N_{f}-(33 / 2)$. For the GLS and Bp sum rules the corresponding Borel transforms coincide ${ }^{3}$ :

$$
\begin{aligned}
\mathrm{B}\left[\mathrm{C}_{\mathrm{Bp}}\right] & =\mathrm{B}\left[\mathrm{C}_{\mathrm{GLS}}\right] \\
& =-\frac{(3+\delta) \exp (5 \delta / 3)}{\left(1-\delta^{2}\right)\left(1-\delta^{2} / 4\right)}
\end{aligned}
$$

The Borel transforms for the Bup and Bp turn out to be closely related 4 , namely

$$
\begin{aligned}
\mathrm{B}\left[\mathrm{C}_{\mathrm{Bup}}\right] & =\left(\frac{2(1+\delta)}{3+\delta}\right) \mathrm{B}\left[\mathrm{C}_{\mathrm{Bp}}\right] \\
& =-\frac{2 \exp (5 \delta / 3)}{(1-\delta)\left(1-\delta^{2} / 4\right)}
\end{aligned}
$$

Notice two similar features of Eq.(5) and Eq.(6): the leading $\delta=1$ poles in the Borel transforms of Eq.(5) and Eq.(6), which are lying on the positive axis of the $\delta$-plane and are called leading infrared renormalons (IRRs), have identical negative residues. Moreover, the residues of $\delta=-1$ poles in the same Borel transforms, called leading ultraviolet renormalons (UVRs), are strongly suppressed in relation to the residues of the leading IRRs ${ }^{4}$. Indeed, in the case of Eq.(5) the suppression factor is $(1 / 2) \exp (-10 / 3) \approx$ 0.018 , while in the case of Eq.(6) it is identically equals to zero.

Eq.(5) indicates the validity of the 1.h.s. of the perturbative Eq.(2) $\mathrm{C}_{\mathrm{GLS}}\left(a_{s}\right) \approx$ $\mathrm{C}_{\mathrm{Bp}}\left(a_{s}\right)$ in the asymptotic regime (for technical details see Ref. 3). This suggests that the "light-by-light"-type contributions to highorder perturbative corrections to $\mathrm{C}_{\mathrm{GLS}}\left(a_{s}\right)$ should be small. The existing order $a_{s}^{3}$ analytical results ${ }^{12}$ confirm this guess and are underly definite next-to-next-to-leading order phenomenological applications of Ref. 13.

The consequence of Eq. (6) is even more clear. In this case the behaviour of asymptotic series for the coefficient function is governed by the same IRR, which dominates the asymptotic perturbative expressions for the coefficient function of the $\mathrm{Bp}$ and GLS sum rules. Therefore the r.h.s. of Eq.(2), namely $\mathrm{C}_{\mathrm{Bp}}\left(a_{s}\right) \approx \mathrm{C}_{\mathrm{Bup}}\left(a_{s}\right)$ is valid as well. Moreover, this explains the similarity between next-to-next-to-leading order perturbative QCD contributions to $\mathrm{C}_{\mathrm{Bp}}\left(a_{s}\right)$ and $\mathrm{C}_{\text {Bup }}\left(a_{s}\right)$ observed in Ref. ${ }^{14}$.

A few words about non-perturbative $1 / Q^{2}$ effects are in order. The IRRs in the Borel transforms for all three sum rules at $\delta=1$ and $\delta=2$ generate ambiguities in the corresponding Borel integrals of Eq.(4). In our analysis we will modify the integration contour by introducing small semi-circles, which are going above these poles in the Borel plane. This PV procedure introduse an extra negative IRR -induced contribution, namely

$$
\Delta \mathrm{C}_{\text {sum rules }} \approx-\frac{32 \exp (5 / 3)}{3 \beta_{0}} \frac{\Lambda \frac{2}{\mathrm{MS}}}{\mathrm{Q}^{2}} .
$$

A similar term was derived in Ref.11 in the context of discussions of the GLS sum rule. This IRR induced power correction should be cancelled by the leading UVR in the coefficient function of the twist- $41 / Q^{2}$-term, as was mentioned in the work of Ref.15 and 
explicitly shown in the theoretical analysis of Ref. 16. However, the negative sign and the identical value of Eq.(7) in the case of all three sum rules can be considered as an argument in favour of negative and identical values of the numerical results for $1 / \mathrm{Q}^{2}$ contributions to different sum rules. This statement is similar to the hypothesis of universality of power corrections and IRR contributions ${ }^{17}$ and supports the validity of Eq.(3). In Table 1 the numerical expressions for the numerators of $1 / \mathrm{Q}^{2}$-corrections calculated within different non-perturbative models are summarized.

Table 1. Coefficients of the twist- 4 contributions to DIS sum rules (in $\mathrm{GeV}^{2}$ )

\begin{tabular}{lccc}
\hline $\mathbf{A}$ & $\mathbf{B}$ & $\mathbf{C}$ & Ref. \\
\hline $0.10 \pm 0.05$ & - & $0.13 \pm 0.07$ & {$\left[{ }^{18}\right]$} \\
- & $0.06 \pm 0.03$ & - & {$\left[{ }^{19}\right]$} \\
$0.16 \pm 0.08$. & $0.22 \pm 0.12$ & $0.16 \pm 0.08$ & {$\left[{ }^{20}\right]$} \\
- & $0.03 \pm 0.01$ & - & {$\left[^{21}\right]$} \\
- & $0.03 \pm 0.06$ & - & {$\left[^{22}\right]$} \\
$0.08 \pm 0.04$ & $0.09 \pm 0.03$ & - & {$\left[{ }^{23}\right]$} \\
- & - & $0.16 \pm 0.08$ & {$\left[{ }^{24}\right]$} \\
- & $0.10 \pm 0.07$ & - & {$\left[{ }^{25}\right]$} \\
\hline
\end{tabular}

The numbers from Refs. 18- 22 were obtained using three-point function QCD sum rules method with different interpolating currents. The results ${ }^{23}$, ${ }^{24}$ were obtained using an instanton model of the nonperturbative vacuum. Within the quoted error bars they all are consistent. However, the work of Ref. 22 demonstrates the importance of careful estimates of theoretical uncertainties and is putting a huge question mark next to the small result of Ref. ${ }^{21}$, which is significantly smaller than the one from Ref.19. Moreover, phenomenological determination of the parameter $\mathrm{B}$ from the polarized structure function data by means of integrating the $h(x) / Q^{2}$ model extracted from the data ${ }^{25}$ supports the result of the QCD sum rules analysis of Ref.19.

Thus the numbers presented in Table
1 and discussions below it lend support to Eq. (3) and together with the perurbative equation of Ref.(2) are consistent with the main relation of Eq.(1) discovered in Ref.1 and discussed in detail in Ref.2. However, more careful checks are still needed. One of them is related to the necessity of independent calculations of the parameters A, B and C. Another one is presumes more detailed studies of the outcomes of taking Borel transform from the Borel images, as calculated in Refs.3, 4. The first results, presented in ${ }^{26}$ for $N_{f}=0$ seem to confirm Eq.(1) in the region $Q^{2} / \Lambda^{2} \geq 6$ which approximately corresponds to the region of energies considered in ${ }^{1},{ }^{2}$.

Consider now the experimental consequences of Eq.(1). We will use experimental values of CCFR-NuTeV collaboration for the $\mathrm{Q}^{2}$-dependence of the GLS sum rule 27 , extract from them the $\mathrm{Q}^{2}$ dependence of the Bp sum rule using the approximate theoretical relation of Eq.(1) and compare these results with the concrete experimental data (see Table 2). One can see that

Table 2. The comparison of Bp results extracted from GLS values using Eq. (1) with the direct experimental extractions of $\mathrm{Bp}$

\begin{tabular}{cccc}
\hline $\mathrm{Q}^{2}$ & Bp from Eq.(1) & \multicolumn{2}{c}{ Bp (expt) } \\
\hline 2.00 & $0.174 \pm 0.012$ & $0.169 \pm 0.025$ & {$\left[{ }^{28}\right]$} \\
3.16 & $0.178 \pm 0.008$ & $0.164 \pm 0.023$ & {$\left[{ }^{28}\right]$} \\
5.01 & $0.195 \pm 0.014$ & $0.181 \pm 0.022$ & {$\left[{ }^{29}\right]$} \\
12.5 & $0.196 \pm 0.016$ & $0.195 \pm 0.029$ & {$\left[{ }^{30}\right]$} \\
\hline
\end{tabular}

within existing error bars the results for Bp sum rule motivated by the GLS sum rule experimental numbers the ones based on the exact experimental measurements are in good agreement. Moreover extracted by theoreticians from existing SLAC and SMC data, $\operatorname{Bp}\left(3 \mathrm{GeV}^{2}\right)=0.177 \pm 0.018{ }^{31}$ which, within error bars, does not contradict the value $\mathrm{Bp}\left(3 \mathrm{GeV}^{2}\right)=0.164 \pm 0.011^{32}$, and is in beautiful ( although presumably accidental) 
agreement with the result of applying Eq.(1) to the experimental value of the GLS sum rule (see second entry in Table 2).

In order to estimate better the errors and limitations of Eq.(1), it is highly desirable to try to extract the $Q^{2}$ dependence of the GLS sum rule from already existing $\mathrm{NuTeV}$ data for $x F_{3}$. It would also be interesting to get new data for the Bp sum rule. These data may be obtained at JLAB and by COMPASS Collaboration at CERN, if it will be able to continue running this experiment using hydrogen target. Another interesting application of Eq.(1) would be the estimation of the $\mathrm{Q}^{2}$ dependence of the still experimentally unmeasured Bup sum rule. This proposal was made whilst planning for the hadronic program of Neutrino Factories ${ }^{33},{ }^{34}$.

I am grateful to I.A. Savin for discussions. It is the pleasure to thank D.J.Broadhurst for fruitful collaboration and the members and visitors to IPPP, Durham, UK, where this write-up of my talk at ICHEP-06 was prepared, for the interest in its content. The work is done within the program of RFBR Grants 05-01-00992 and 0602-16659.

\section{References}

1. A. L. Kataev, JETP. Lett. 81, 608 (2005).

2. A. L. Kataev, Mod. Phys. Lett. A20, 2007 (2005).

3. D. J. Broadhurst and A. L. Kataev, Phys. Lett. B315, 179 (1993).

4. D. J. Broadhurst and A. L. Kataev, Phys. Lett. B544, 154 (2002).

5. I. Hinchliffe and A. Kwiatkowski, Ann. Rev. Nucl. Part. Sci. 46, 609 (1996).

6. E. V. Shuryak and A. I. Vainshtein, Nucl. Phys. B199, 451 (1982).

7. E. V. Shuryak and A. I. Vainshtein, Nucl. Phys. B201, 141 (1982).

8. X. D. Ji and P. Unrau, Phys. Lett. B333, 228 (1994).

9. V. I. Zakharov, Nucl. Phys. B 385, 452 (1992).

10. M. Beneke, Phys. Rept 317, 1 (1999).

11. M. Beneke and V. M. Braun, in Handbook of $Q C D$, ed. M. Shifman (World Scientific Singapore, 2001).

12. S. A. Larin and J. A. M. Vermaseren, Phys Lett B259, 345 (1991).

13. S. J. Brodsky, G. T. Gabadadze, A. L. Kataev and H. J. Lu, Phys. Lett. B372, 133 (1996).

14. E. Gardi and M. Karliner, Nucl. Phys 529, 383 (1998).

15. A. H. Mueller, Phys. Lett. B308, 355 (1993).

16. E. Gardi and R. G. Roberts, Nucl.Phys B 653, 227 (2003).

17. Y L. Dokshitzer, G. Marchesini and B. R. Webber, Nucl. Phys. B469, 93 (1996)

18. V. M. Braun and A. V. Kolesnichenko, Nucl.Phys B283, 723 (1987).

19. I. I. Balitsky, V. M. Braun and A. V. Kolesnichenko, Phys. Lett B242, 245 (1990); Erratum:Phys. Lett. B318, 648 (1993).

20. G. G. Ross and R. G. Roberts, Phys. Lett. B322, 425 (1994).

21. E. Stein at. al., Phys. Lett. B343, 369 (1995).

22. B. L. Ioffe, Phys.Atom.Nucl 60, 1707 (1997).

23. J. Balla, M. V. Polyakov and C. Weiss, Nucl. Phys. B510, 327 (1998).

24. C. Weiss, J. Phys. G29, 1981 (2003).

25. A. V. Sidorov and C. Weiss, Phys.Rev. D73, 074016 (2006) .

26. P. M. Brooks and C. J. Maxwell, Phys.Rev. D74, 065012 (2006); C.J. Maxwell, talk at ICHEP-06 Conference, Moscow, 2006.

27. J. H. Kim et al., Phys. Rev. Lett. 81, 3595 (1998).

28. K. Abe et al. [E143 collaboration], Phys. Rev. D58, 112003 (1998).

29. B. Adeva et al. [Spin Muon Collaboration], Phys.Rev. D 58, 112002 (1998).

30. B. Adeva et al. [Spin Muon Collaboration (SMC)], Phys. Lett B 412, 414 (1997).

31. G. Altarelli, R. D. Ball, S. Forte and G. Ridolfi, Nucl. Phys. Bf 496, 337 (1997).

32. J. R. Ellis, E. Gardi, M. Karliner and M. A. Samuel, Phys. Lett. B366, 268 (1996).

33. M. L. Mangano et al.,ECFA/CERN studies of a European neutrino factory ed. A. Blondel et all. CERN Yellow Report CERN-2004-002 185, (2004); hep-ph/0105155.

34. S. I. Alekhin and A. L. Kataev, J. Phys. G 29, 1993 (2003) 